\title{
CARACTERÍSTICAS EDÁFICAS RELACIONADAS À PRODUÇÃO DE UM POVOAMENTO DE Araucaria angustifolia (Bert.) O. Kuntze
}

\author{
Wagner Correa Santos ${ }^{1}$, Nelson Carlos Rosot ${ }^{2}$, Maria Augusta Doetzer Rosot ${ }^{3}$ \\ ${ }^{1}$ Eng. Florestal, M.Sc., Colégio Florestal de Irati, Irati, PR, Brasil - correasantos@gmail.com \\ ${ }^{2}$ Eng. Florestal, Dr., Depto. de Ciências Florestais, UFPR, Curitiba, PR, Brasil - ncrosot@ufpr.br \\ ${ }^{3}$ Eng $^{\mathrm{a}}$. Florestal, Dr ${ }^{\mathrm{a}}$., Embrapa Florestas, Colombo, PR, Brasil - augusta@cnpf.embrapa.br \\ Recebido para publicação: 04/04/2008 - Aceito para publicação: 20/05/2009
}

\begin{abstract}
Resumo
Este trabalho teve por objetivo estudar a influência dos fatores edáficos na produção de 12 procedências de Araucaria angustifolia (pinheiro-do-paraná) na Estação Experimental de Rio Negro da Universidade Federal do Paraná (UFPR). O experimento consiste em 36 parcelas de aproximadamente $550 \mathrm{~m}^{2}$ cada, instaladas em blocos ao acaso, com 3 repetições para cada procedência. Foram feitas avaliações de diâmetro à altura do peito (DAP), altura dominante $\left(\mathrm{H}_{\mathrm{dom}}\right)$, área basal $(\mathrm{G} / \mathrm{ha})$ e volume por hectare $(\mathrm{V} / \mathrm{ha})$ de 24 árvores distribuídas em todas as classes diamétricas, e analisados a profundidade e os atributos físicos e químicos de solo. Constatou-se que a diferença de produtividade está ligada aos fatores edáficos e não às procedências testadas. Assim, foi necessário agrupar as parcelas em classes homogêneas de solo, usando as ferramentas estatísticas multivariadas de análise de agrupamento e discriminante. Foram determinados cinco grupos de parcelas com classes semelhantes de solo, sendo que a variável "profundidade" apresentou o melhor valor discriminante. Analisando a relação entre os grupos de solos formados e os valores dendrométricos $\mathrm{H}_{\mathrm{dom}}$, G/ha e V/ha, pôde-se verificar que para $\mathrm{H}_{\mathrm{dom}}$ não existiram diferenças significativas entre os grupos de solos, e que apenas um grupo apresentou valores significativamente inferiores aos demais para G/ha e V/ha. Dessa forma, constatou-se que, no experimento, há dois sítios distintos, e a profundidade maior ou menor que um metro de solo define em qual sítio cada parcela está contida.
\end{abstract}

Palavras-chave: Solo; teste de procedências; sítio.

\begin{abstract}
Edaphic aspects related to the yield of an Araucaria angustifolia (Bert.) O. Kuntze stand. This work aimed at the study of the influence of edaphic factors on the yield of 12 different provenances of Araucaria angustifolia (parana-pine) at the Rio Negro Experimental Station - Federal University of Paraná (UFPR). The experiment consists of 36 plots of 550 sq.m. each, laid out in a randomized block design with three replicates by provenance, where the following data have been collected: d.b.h., dominant height $\left(\mathrm{H}_{\mathrm{dom}}\right)$, soil depth, soil samples and volume of 24 trees distributed in all diameter classes. No significant differences were detected among plots, thus suggesting that edaphic factors have more influence on the productivity than genetic factors related to seed provenances. Multivariate analysis techniques were used to group plots according to soil chemical and physical characteristics. Five homogenous soil groups were defined and the variable "soil depth" presented the most discriminating power. An analysis of the relationship between soil groups and stand variables showed that, for dominant height, there were no differences between plots associated with different soil groups. For basal area and volume per hectare, only one group was considered different from the others. This fact led to the conclusion that there are two different sites in the experimental area, defined by soil depths lower or higher than 1 meter, respectively.

Keywords: Soils; provenance test; site.
\end{abstract}

\section{INTRODUÇÃO}

A escassez de madeira no mercado brasileiro e as pressões que sofrem as empresas consumidoras dessa matéria-prima, por parte de diversos setores da sociedade, no que se refere às 
questões ambientais, requerem dos profissionais do setor florestal soluções efetivas para a obtenção de produção sustentável com minimização de impactos ambientais.

As espécies nativas representam a minoria das plantações florestais, sendo preteridas em relação às espécies exóticas de crescimento rápido e maior produtividade. No entanto, o desenvolvimento de uma silvicultura voltada às espécies nativas tem potencial para compor florestas de proteção, área de preservação permanente, reserva legal, florestas de uso múltiplo e sistemas agroflorestais. Esses ecossistemas florestais representam uma alternativa para a conservação da biodiversidade de fauna e flora e garantem a produção de madeira com qualidades específicas, que as espécies de crescimento rápido não possuem. Como exemplos, podem ser citados móveis finos, construção civil e instrumentos musicais.

Araucaria angustifolia, ou pinheiro-do-paraná, apresenta um grande potencial na sua região de ocorrência natural, porém é pouco conhecida em termos de crescimento, de produção e dos fatores que os influenciam, como a qualidade do material genético, a qualidade do sítio e a silvicultura (densidade de plantio, limpeza, poda e desbaste).

O pinheiro-do-paraná foi explorado sem nenhuma preocupação desde o século XIX, tendo sido o corte de caráter extrativista, destinado ao preparo de áreas para o cultivo agrícola, um dos fatores responsáveis pela diminuição das reservas de florestas naturais na sua região de ocorrência (FUPEF, 1978).

A implantação de florestas comerciais nessas e outras áreas ocorreu, principalmente, a partir da década de 60, com o início dos incentivos fiscais, tendo sido usadas espécies de crescimento rápido, como Pinus spp. e Eucalyptus spp., que proporcionavam maior rentabilidade financeira. Os plantios de pinheiro-do-paraná, até o fim da década de 70, apresentavam uma área de aproximadamente de 45.000 ha no estado do Paraná, ou apenas cerca de 7\% de toda área reflorestada (BREPOHL, 1980).

Os poucos plantios comerciais de pinheiro-do-paraná e a drástica redução da taxa de implantação a partir da década de 80 limitaram o desenvolvimento de novas pesquisas e tecnologia para essa espécie. Ainda hoje observa-se que os plantios remanescentes encontrados não são, em sua grande maioria, manejados, não sofrem tratos culturais e sua produção está estagnada, causando prejuízos e proporcionando uma visão que não corresponde ao potencial real e à viabilidade de plantios comerciais.

A não consideração dos fatores limitantes que determinam a capacidade produtiva do pinheirodo-paraná fez com que a espécie fosse caracterizada por apresentar baixos incrementos. Porém, Carvalho (2003) comenta que apenas $25 \%$ da área de ocupação natural são economicamente viáveis para plantios.

Atualmente, a Portaria $n^{\circ} 046$, de 20 de março de 2006, complementada pela portaria $\mathrm{n}^{\circ} 060$, de abril de 2006, do Instituto Ambiental do Paraná (IAP), estabelece critérios para o corte de espécies florestais consideradas em extinção no estado do Paraná, entre as quais se inclui o pinheiro-do-paraná, proibindo o corte raso de florestas secundárias em estágio inicial de sucessão em áreas maiores que 5 ha e $\mathrm{o}$ aproveitamento de madeira superior a $15 \mathrm{~m}^{3} \mathrm{em}$ toras.

O desenvolvimento de um sistema de manejo adequado aos povoamentos de pinheiro-do-paraná, aliado à identificação dos fatores que limitam o crescimento e produção, pode contribuir para o aumento do interesse de seu uso em plantios comerciais, possibilitando a retomada da sua competitividade no mercado e assegurando a sustentabilidade por meio de equilíbrio entre a produção e conservação do meio ambiente.

O presente trabalho, executado em um teste de procedência de Araucaria angustifolia na Fazenda Experimental de Rio Negro da UFPR, teve como objetivos avaliar a influência das propriedades de solo de forma isolada e combinada sobre a produção dessa espécie e elaborar e comparar a classificação dendrométrica e ecológica para as classes de sítios encontradas na superfície do experimento.

\section{REVISÃO BIBLIOGRÁFICA}

\section{Silvicultura do pinheiro-do-paraná}

Araucaria angustifolia pertence à família Araucariaceae, sendo conhecida por pinheiro-doparaná, pinho ou pinheiro brasileiro, entre outras denominações. Ocorre desde o estado do Rio Grande do Sul até Minas Gerais, em altitudes superiores a 900 metros, sendo que nos estados do sul do Brasil ocorre em regiões acima de $500 \mathrm{~m}$ de altitude (LORENZI, 1992). 
Os primeiros plantios de pinheiro-do-paraná em uma escala maior foram iniciados a partir de 1920, na Companhia de Melhoramento de São Paulo, no município de Caieiras. A partir de 1945, o Instituto Nacional do Pinho e empresas privadas, como a Klabin Celulose e Papel S/A, começaram a desenvolver plantios em escalas ainda maiores, atingindo o auge com o surgimento dos incentivos fiscais na década de 60 (BREPOHL, 1980).

Os plantios de pinheiro-do-paraná tiveram um declínio devido aos problemas ecológicos da espécie, como a alta exigência por solos produtivos, e por fatores de mercado, como a forte concorrência das espécies exóticas, menos exigentes em relação a fatores edáficos e com períodos mais curtos de rotação (DIETRICH, 1979).

Diversos estudos visando obter um melhor método silvicultural e um melhoramento genético da espécie foram apresentados até a década de 80 (GURGEL FILHO, 1980), sendo que, a partir desse período, tornaram-se cada vez mais escassos.

\section{Classificação de sítios}

A capacidade produtiva de uma floresta em uma determinada área é influenciada principalmente por fatores biológicos, edáficos, climáticos e topográficos (PRODAN et al., 1997), e a sua soma e interação define a variável sítio para uma determinada espécie (SPURR, 1952).

Existem dois métodos que podem ser empregados para a realização da classificação de sítio:

(1) Método dendrométrico, que realiza a classificação dos sítios a partir da capacidade produtiva de cada unidade, expressa por uma variável mensurável (altura dominante, por exemplo).

(2) Método ecológico, que envolve fatores físicos, químicos do solo, topográficos e climáticos da região estudada (SALLE, 1993).

\section{Relação entre solo e crescimento do pinheiro-do-paraná}

Diversos fatores afetam a produtividade do pinheiro-do-paraná, e o conhecimento do seu grau de influência possibilita buscar condições para melhorar a produtividade da espécie. A relação entre as características solo e crescimento é considerada um dos fatores limitantes, e o estudo mais aprofundado dessa relação é essencial para aumentar a produtividade da espécie (CASSOL, 1982).

O efeito mais importante da deficiência mineral do solo é a redução do ritmo de crescimento das plantas (SIMÕES, 1972), porém Cassol (1982), Hoppe (1980) e Dietrich (1979) constataram que as características químicas do solo obtidas em laboratório não representam os melhores parâmetros para explicar a variação do crescimento e produtividade de Araucaria angustifolia. A baixa correlação entre o crescimento das árvores e o resultado da análise química de solo deve-se ao fato de que esta indica apenas as concentrações dos nutrientes que, teoricamente, estariam disponíveis para a planta em apenas um estrato do solo, não constituindo um indicador seguro da disponibilidade de nutrientes, devido à grande profundidade das raízes (SIMÕES, 1972). Essa afirmação é corroborada por Cassol (1982), ao ressaltar que a profundidade do solo, associada a outras características físicas, como uma boa porosidade, por exemplo, possibilita a utilização de uma maior área para a penetração das raízes, desenvolvendo-se uma raiz pivotante que pode atingir até $4 \mathrm{~m}$ de profundidade. Dessa forma, a planta pode buscar uma maior quantidade de nutrientes disponíveis no solo, mesmo que este tenha um teor nutricional não significativo.

Hoppe (1980) constatou que as propriedades físicas do solo apresentaram maior influência no crescimento e produção de Araucaria angustifolia quando comparadas às propriedades químicas. As correlações negativas apresentadas entre as propriedades químicas do solo e o crescimento e produção da espécie são consequência do fato de o estudo ter sido desenvolvido em área de Latossolos. No entanto, pelo fato de serem solos minerais, não-hidromórficos, profundos (normalmente superiores a $2 \mathrm{~m}$ ), apresentando horizontes B muito espessos $(>50 \mathrm{~cm})$ e sequência de horizontes $\mathrm{A}, \mathrm{B}$ e $\mathrm{C}$ pouco diferenciados (AGÊNCIA DE INFORMAÇÃO EMBRAPA, 2009), as características físicas dos Latossolos proporcionam um melhor desenvolvimento radicular, devido a uma boa porosidade que, por sua vez, promove uma maior atividade biológica.

Van Goor (1965) afirma que a profundidade do solo é um fator limitante para o crescimento de Araucaria angustifolia, sendo necessário um mínimo de $70 \mathrm{~cm}$ a $100 \mathrm{~cm}$ para um bom crescimento. Esse resultado é confirmado por Cassol (1982), De Hoogh; Dietrich (1979), que obtiveram maiores crescimentos da espécie em solos profundos, acima de $1 \mathrm{~m}$ de profundidade. 
A exigência da espécie por solos profundos e uma alta fertilidade foi constatada no estado do Paraná por Carvalho (2003), registrando plantios que apresentaram incrementos anuais variando de $1 \mathrm{~m}^{3} \cdot \mathrm{ha}^{-1}$ a $26 \mathrm{~m}^{3} \mathrm{ha}^{-1}$, o que corrobora que um dos aspectos mais problemáticos do pinheiro-do-paraná como espécie para reflorestamento é, realmente, a sua exigência em solos.

\section{MATERIAL E MÉTODOS}

\section{Caracterização da área de estudo}

A área de estudo consiste em um teste de procedência de Araucaria angustifolia instalado em uma área de 2 ha na Estação Experimental de Rio Negro da Universidade Federal do Paraná, situada no município de Rio Negro, PR.

Maack (1981) define o clima da região como tipo Cfb (subtropical quente temperado), com precipitação média anual em torno de $1300,5 \mathrm{~mm}$ bem distribuídos ao longo do ano. A temperatura média anual varia de $12^{\circ} \mathrm{C} \mathrm{a} 18{ }^{\circ} \mathrm{C}$, com frequentes geadas no inverno e altitude acima de 800 metros.

No ano de 1967, foram instaladas 36 parcelas para um teste de 12 procedências de Araucaria angustifolia, usando-se o delineamento experimental em blocos ao acaso com três repetições para cada procedência. As procedências testadas foram: Guapiara (SP), Telêmaco Borba (PR), Pitanga (PR), Imbituva (PR), Laranjeiras do Sul (PR), Rio Negro (PR), União da Vitória (PR), Ponte Serrada (SC), Ponte Alta do Sul (SC), Vacaria (RS), Francisco Beltrão (PR) e Cascavel (PR) (Figura 1).

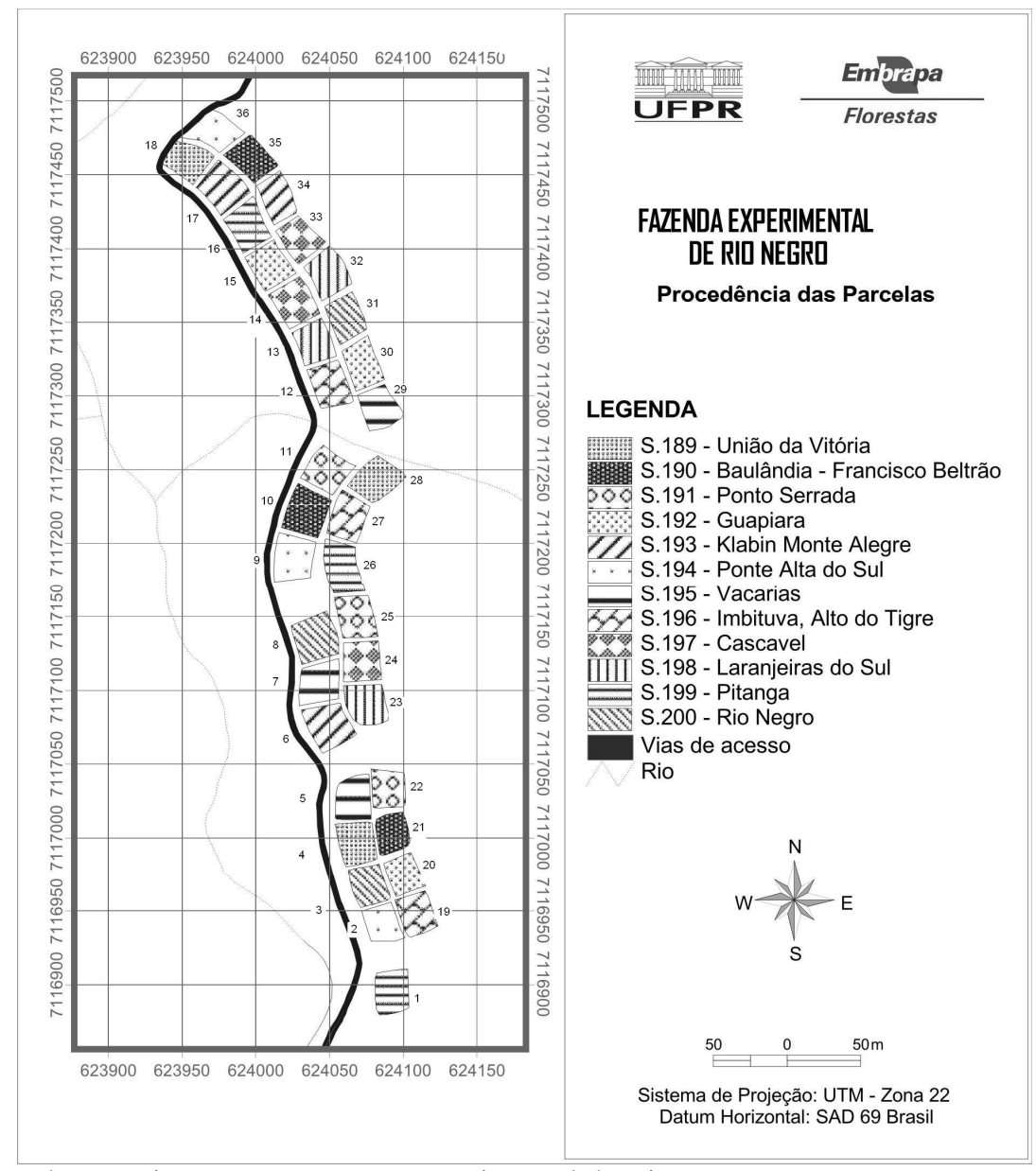

Figura 1. Área do experimento na Estação Experimental de Rio Negro.

Figure 1. Study area located at Rio Negro Experimental Station. 


\section{Coleta de dados e procedimento de laboratório}

Cada parcela contém sete linhas de 13 plantas, no espaçamento médio de $3 \mathrm{~m}$ x $1,90 \mathrm{~m}$. A parcela útil ficou constituída pelas 55 árvores centrais.

Foram medidos os perímetros das 36 parcelas para obtenção de suas áreas reais, uma vez que o experimento está instalado em curva de nível e, portanto, com áreas variáveis por parcela. Mediu-se o DAP de todas as árvores e a altura das cinco árvores mais grossas por hectare (altura dominante).

As árvores a serem derrubadas para a obtenção do volume foram selecionadas a partir da distribuição diamétrica ajustada com os dados de DAP. Cada classe diamétrica foi definida com uma amplitude de um (1) desvio padrão em relação ao DAP médio, estabelecendo-se três classes de desvio padrão acima do valor médio e três classes abaixo desse valor. Foram derrubadas quatro árvores por classe diamétrica, totalizando 24 árvores, pelo fato de a classe 1 (DAP -3s) não apresentar nenhum representante.

Amostras de solo foram coletadas a uma profundidade de $0-20 \mathrm{~cm}$, sendo retiradas cinco subamostras, que formaram uma amostra composta para cada parcela do experimento. Utilizou-se um trado holandês de $1,80 \mathrm{~m}$ para verificar a profundidade do solo e também foi retirada uma amostra não deformada do solo em um anel volumétrico.

As amostras foram enviadas ao Laboratório de Solos da Embrapa Florestas no município de Colombo, PR, para análise química e física.

Para a análise química, foram obtidos dados de acidez ativa $(\mathrm{pH}) \mathrm{em} \mathrm{CaCl}_{2}$, macronutrientes como fósforo $(\mathrm{P})$, potássio $(\mathrm{K})$, cálcio $(\mathrm{Ca})$, magnésio $(\mathrm{Mg})$ e nitrogênio em $\mathrm{cmolc}^{-\mathrm{dm}^{-3}}$, micronutrientes como ferro $(\mathrm{Fe})$, cobre $(\mathrm{Cu})$, manganês $(\mathrm{Mn})$ e zinco $(\mathrm{Zn})$ em $\mathrm{MG}_{\mathrm{dm}}{ }^{-3}$ e outros nutrientes importantes para o desenvolvimento da planta, como o sódio (Na) em mg.dm ${ }^{-3}$ e alumínio (A $)$ em cmolc. $\mathrm{dm}^{-3}$.

$\mathrm{Na}$ análise das variáveis morfológicas do solo, a profundidade (em centímetros) em cada parcela foi medida com trado holandês até uma profundidade de 1,80 m, e a declividade (em percentagem), com o auxílio de um clinômetro.

$\mathrm{Na}$ análise física, foram obtidos a densidade do solo em $\mathrm{kg} \cdot \mathrm{dm}^{-3}$, a umidade em g.g $\mathrm{g}^{-1} \mathrm{e} \mathrm{cm}^{3} \cdot \mathrm{cm}^{-3}$, a porosidade total em g. $\mathrm{g}^{-1} \mathrm{e} \mathrm{cm}^{3} . \mathrm{cm}^{-3}$, a macroporosidade em g.g e cm $\mathrm{cm}^{3} \cdot \mathrm{cm}^{-3}$, a microporosidade em g. $\mathrm{g}^{-1}$ $\mathrm{e} \mathrm{cm}^{3} \cdot \mathrm{cm}^{-3}$ e dados de textura em percentagem de areia grossa, areia fina, silte e argila.

\section{Processamento e análise estatística dos dados}

Para cada parcela, foram obtidos os dados de área transversal (g), área basal $(\mathrm{G})$, altura $(\mathrm{H})$, altura dominante $\left(\mathrm{H}_{\mathrm{dom}}\right)$ e volume $(\mathrm{V})$. Para a obtenção das variáveis $\mathrm{H}$ e V, foram ajustados modelos matemáticos de relação hipsométrica e equações de volume, respectivamente.

Para uma classificação dendrométrica do sítio, fez-se necessária uma análise de $\mathrm{H}_{\mathrm{dom}}, \mathrm{G}$ e V das procedências e parcelas, com o objetivo de verificar se a diferença de produtividade do experimento estava relacionada à qualidade e adaptação das sementes (procedências) ou a outros fatores presentes nas parcelas individuais. Para isso, usou-se a técnica de análise de variância com subamostragem e o teste comparativo de médias de Tukey.

$\mathrm{Na}$ análise de variância com subamostragem, as procedências foram consideradas como tratamento, a média das alturas dominantes, área transversal e volume de cada parcela como repetição e as medidas das árvores individuais nas parcelas como sub-repetições. Assim, obteve-se um total de 12 tratamentos, 36 repetições e 180 sub-repetições para altura dominante e 1980 sub-repetições para área transversal e volume.

Para a determinação ecológica do sítio, foi usada a ferramenta de análise de correlação de Pearson, que determina o grau de associação entre duas variáveis, além de técnicas de estatística multivariada (análise de agrupamento e análise discriminante).

$\mathrm{Na}$ análise de correlação de Pearson, os dados de análise química, física e morfológica dos solos foram correlacionados com a altura dominante, DAP, altura média, área basal e volume das parcelas.

Também foram empregadas técnicas de análise multivariada em função da necessidade de se analisar conjuntamente um grande número de variáveis de solo. A análise de grupamento possibilitou a geração de classes a partir das características contidas na matriz de dados, ou seja, foram formados grupos de parcelas semelhantes a partir de análise de solo, com base nas propriedades químicas, físicas e morfológicas. O método de classificação empregado foi o Aglomerativo Hierárquico, que considera uma série de fusões sucessivas até esgotar-se a possibilidade de combinação. 
Para determinar que principais variáveis do solo tiveram maior peso na formação dos grupos formados, empregou-se a técnica de análise discriminante com o método STEPWISE.

\section{RESULTADOS E DISCUSSÃO}

\section{Avaliação dendrométrica}

A ANOVA com subamostragem para variável altura dominante (Tabela 1) mostrou não existir diferenças significativas entre tratamentos (procedências) para uma probabilidade de $95 \%$. No entanto, considerando as repetições dentro do tratamento para uma probabilidade de $95 \%$, pode-se afirmar que a altura dominante de pelo menos uma parcela é estatisticamente diferente das demais.

Tabela 1. ANOVA com subamostragem para altura dominante (m).

Table 1. ANOVA with subsampling for dominant height $(\mathrm{m})$.

\begin{tabular}{lccccc}
\hline ANOVA & GL & SQ & QM & F & F tab (0,05) \\
\hline Entre parcelas (repetições) & 35 & 358,8784 & & & \\
Entre procedências (tratamento) & 11 & 157,5011 & 14,31828 & 1,706442 & 2,216311 \\
Entre repetições dentro do tratamento & 24 & 201,3773 & 8,390722 & 6,876702 & 1,593229 \\
Entre árvores dentro da parcela & 144 & 175,704 & 1,220167 & & \\
\hline Total & 179 & 534,5824 & & & \\
\hline
\end{tabular}

Para área transversal, constataram-se resultados semelhantes aos encontrados para a variável altura dominante, não se detectando diferenças significativas entre as procedências, e sim diferença de área transversal entre parcelas (Tabela 2).

Tabela 2. ANOVA com subamostragem para área transversal $\left(\mathrm{m}^{2}\right)$.

Table 2. ANOVA with subsampling for cross-sectional area (sq.m).

\begin{tabular}{lccccc}
\hline ANOVA & GL & SQ & QM & F & F tab (0,05) \\
\hline Entre parcelas & 35 & 0,04280 & & & \\
Tratamentos & 11 & 0,01471 & 0,001337 & 1,142897 & 2,216311 \\
Entre repetições dentro do tratamento & 24 & 0,02809 & 0,00117 & 1,598894 & 1,522917 \\
Entre árvores dentro das parcelas & 1944 & 1,42285 & 0,000732 & & \\
\hline Total & 1979 & 1,46565 & & & \\
\hline
\end{tabular}

Para a variável volume (Tabela 3), constatou-se não existir diferenças significativas entre procedências e parcelas.

Tabela 3. ANOVA com subamostragem para volume $\left(\mathrm{m}^{3}\right)$.

Table 3. ANOVA with subsampling for volume (cu.m).

\begin{tabular}{lccccc}
\hline ANOVA & GL & SQ & QM & F & F tab (0,05) \\
\hline Entre parcelas & 35 & 4,47808 & & & \\
Tratamentos & 11 & 1,67617 & 0,152379 & 1,305215 & 2,216311 \\
Entre repetições dentro do tratamento & 24 & 2,80191 & 0,116746 & 1,447023 & 1,522917 \\
Entre árvores dentro das parcelas & 1944 & 156,84270 & 0,08068 & & \\
\hline Total & 1979 & 161,32079 & & & \\
\hline
\end{tabular}

Os resultados para a variável altura dominante, área transversal e volume mostraram a inexistência de diferenças estatísticas entre as procedências, o que sugere que a variabilidade genética não influenciou a produtividade do experimento. Esse resultado vem se contrapor aos obtidos por diversos autores, como Baldanzi; Araújo (1971), que constataram diferenças entre procedências para o mesmo local de estudo aos três anos de idade. Por outro lado, Gurgel et al. (1965), Fahler; Di Luca (1980), Kageyama; Jacob (1980), Monteiro; Spelz (1980), Shimizu; Higa (1980) e Alves (1985) obtiveram o mesmo resultado, ou seja, detectaram diferenças significativas entre procedências em experimentos localizados em toda a área de ocorrência natural do pinheiro-do-paraná. 
As diferenças entre procedências provavelmente são devidas ao fato de os estudos terem sido executados em idades iniciais, em sua grande maioria antes dos 10 anos de idade, podendo cada procedência apresentar taxas de crescimento diferentes e, consequentemente, atingindo sua capacidade máxima de produção em períodos diferentes. Posteriormente, no entanto, pode-se obter produtividades semelhantes em um mesmo sítio.

Considerando não mais as procedências, mas as parcelas, pôde-se constatar que, para as variáveis altura dominante e área transversal, existe diferença entre parcelas, não sendo constatado o mesmo para a variável volume. Sabendo-se que a altura dominante é a variável que expressa a qualidade de sítio, pode-se afirmar que a variação da capacidade produtiva do experimento não está ligada às procedências das sementes, e sim à localização das parcelas no local do experimento. Considerando-se o fator clima constante, a variação de sítio do experimento poderia, então, estar ligada, principalmente, ao fator edáfico.

\section{Avaliação ecológica do sítio}

A tabela 4 mostra os resultados obtidos na análise de correlação de Pearson entre as variáveis de solo e as variáveis dendrométricas. Pode-se constatar que os dados morfológicos explicam, em média, 41,6\% da variação das variáveis dendrométricas. Já as variáveis químicas e físicas do solo explicam apenas $19,15 \%$ e $19,4 \%$, respectivamente.

A variável altura dominante apresentou menor correlação em relação às variáveis de solo se comparada às outras medidas tomadas (média de 15,6\%), sendo 40,9\% para morfológicas, $18,1 \%$ e 10,5\% para as químicas e físicas, respectivamente.

A partir desses resultados, verifica-se uma baixa correlação entre as características do solo e as variáveis dendrométricas, tendo as características morfológicas (profundidade e declividade) se destacado em relação às demais.

Tabela 4. Correlação em percentagem entre as variáveis dendrométricas e variáveis do solo.

Table 4. Correlation (in percentage) between dendrometric and edaphic variables.

\begin{tabular}{lcccc}
\hline Variáveis & \multicolumn{4}{c}{ Variáveis do solo } \\
\cline { 2 - 5 } dendrométricas & Morfológicas & Físicas & Químicas & Média \\
\hline Dap $(\mathrm{cm})$ & $44,8 \%$ & $22,8 \%$ & $21,6 \%$ & $23,6 \%$ \\
$\mathrm{H}_{\mathrm{dom}}(\mathrm{m})$ & $40,9 \%$ & $18,1 \%$ & $10,5 \%$ & $15,6 \%$ \\
$\mathrm{G} / \mathrm{ha}\left(\mathrm{m}^{2} / \mathrm{ha}\right)$ & $38,4 \%$ & $16,5 \%$ & $20,1 \%$ & $19,8 \%$ \\
Altura $(\mathrm{m})$ & $45,0 \%$ & $22,8 \%$ & $22,7 \%$ & $24,2 \%$ \\
Volume $\left(\mathrm{m}^{3} / \mathrm{ha}\right)$ & $38,9 \%$ & $16,6 \%$ & $20,4 \%$ & $24,2 \%$ \\
\hline Média & $41,6 \%$ & $19,4 \%$ & $19,1 \%$ & $21,5 \%$ \\
\hline
\end{tabular}

O resultado da análise de agrupamento é mostrado no dendrograma na figura 2, sendo a distância euclidiana relativa (medida de similaridade) mostrada no eixo $\mathrm{Y}$ e as parcelas no eixo $\mathrm{X}$.

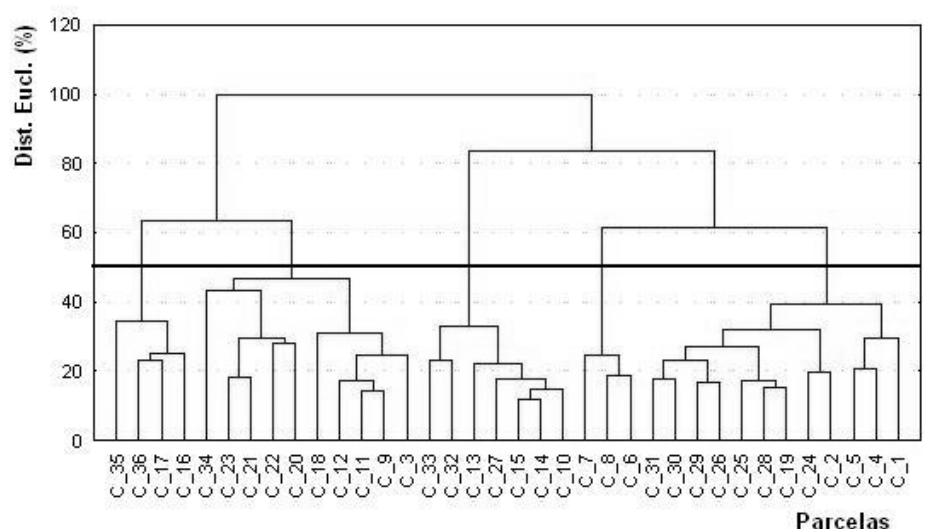

Figura 2. Dendrograma da análise de agrupamento (método hierárquico).

Figure 2. Cluster analysis dendrogram (hierarchical method). 
A linha de formação de grupos foi traçada a 50\% da amplitude das distâncias euclidianas, gerando-se cinco grupos distintos de parcelas que apresentam características estatisticamente semelhantes de solo dentro do grupo. A qualidade desse agrupamento pôde ser constatada em campo, tendo-se observado que as parcelas 6,7 e 8 são qualitativamente inferiores às demais. Nesta análise, elas formaram um grupo distinto.

Analisando espacialmente no terreno os grupos formados, verificou-se que parcelas fisicamente próximas foram lotadas em grupos semelhantes, podendo-se, assim, constatar manchas de solos com as mesmas características (Figura 3).

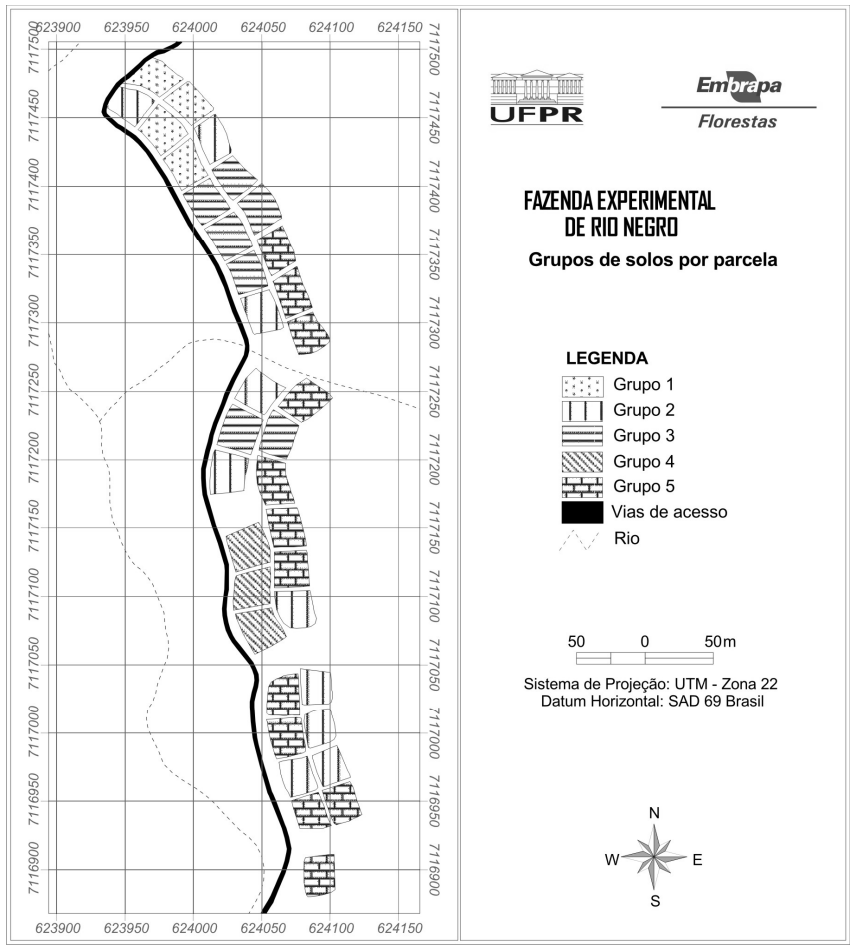

Figura 3. Mapa dos grupos de solos.

Figure 3. Map of soil groups.

Com os grupos já definidos pela análise de agrupamento, a verificação de quais variáveis do solo tiveram uma maior significância na sua formação foi executada usando-se a ferramenta estatística multivariada de análise discriminante. Essa análise foi realizada a partir dos cinco grupos formados pela análise de agrupamento. Como fonte de dados, foi usada a matriz de dados de solo, incluindo-se uma coluna denominada "sítio", na qual cada parcela recebeu o número do grupo determinado na análise anterior.

O método de Stepwise selecionou seis variáveis discriminantes, considerando-se um valor de $\mathrm{F}$ crítico igual a 1, com as estatísticas do teste de seleção mostradas na tabela 5.

Tabela 5. Seleção das variáveis discriminantes (método Stepwise).

Table 5. Selection of discriminant variables (Stepwise method).

\begin{tabular}{lccc}
\hline Passo & Variável & F & Lambda de Wilk \\
\hline 1 & Profundidade & 18,042 & 0,3005 \\
2 & Densidade & 12,572 & 0,1123 \\
3 & Macroporosidade & 10,556 & 0,0457 \\
4 & Areia fina & 9,409 & 0,0195 \\
5 & Nitrogênio & 4,860 & 0,0113 \\
6 & Cálcio & 3,438 & 0,0074 \\
\hline
\end{tabular}


A determinação das funções discriminantes foi importante para analisar a contribuição das variáveis. A tabela 6 mostra que as variáveis mais importantes na primeira função foi a densidade, seguida da macroporosidade, na segunda função foram densidade e areia fina, na terceira foram macroporosidade e densidade, na quarta foram profundidade e densidade e na quinta foram densidade e areia fina.

Tabela 6. Funções discriminantes para as variáveis selecionadas.

Table 6. Discriminant functions for the selected variables.

\begin{tabular}{lccccc}
\hline \multirow{2}{*}{ Variáveis } & \multicolumn{5}{c}{ Funções discriminantes } \\
\cline { 2 - 6 } & $\mathbf{1}$ & $\mathbf{2}$ & $\mathbf{3}$ & $\mathbf{4}$ & $\mathbf{5}$ \\
\hline Profundidade & 2,414 & 1,410 & $-1,745$ & $-10,933$ & 1,771 \\
Densidade & $-11,557$ & $-3,560$ & 2,968 & 7,635 & 3,179 \\
Macroporosidade & $-8,200$ & $-0,935$ & 4,866 & 3,601 & $-0,227$ \\
Areia fina & $-1,960$ & $-1,487$ & $-0,290$ & $-3,738$ & 2,997 \\
Nitrogênio & 4,698 & 0,415 & $-2,917$ & $-6,438$ & 1,399 \\
Cálcio & $-2,059$ & 0,057 & $-1,127$ & $-0,704$ & 1,473 \\
Constante & $-15,891$ & $-3,032$ & $-5,584$ & $-24,155$ & $-3,754$ \\
\hline
\end{tabular}

A tabela 7 mostra os percentuais de classificação correta e incorreta dos casos, podendo-se assim confirmar a precisão da técnica de agrupamento. Observou-se que as cinco funções discriminantes podem ser utilizadas para alocar novos indivíduos dentro dos grupos, constatando-se que nos cinco grupos formados todas as parcelas foram classificadas corretamente.

Tabela 7. Resultado da classificação das amostras na análise discriminante.

Table 7. Results of sample classification in discriminant analysis.

\begin{tabular}{lcccccc}
\hline \multirow{2}{*}{ Grupos } & \multirow{2}{*}{ Classificação (\%) } & $\mathbf{1}$ & $\mathbf{2}$ & $\mathbf{3}$ & $\mathbf{4}$ & $\mathbf{5}$ \\
\cline { 2 - 7 } & 100 & 4 & 0 & 0 & 0 & 0 \\
1 & 100 & 0 & 10 & 0 & 0 & 0 \\
2 & 100 & 0 & 0 & 7 & 0 & 0 \\
3 & 100 & 0 & 0 & 0 & 3 & 0 \\
4 & 100 & 0 & 0 & 0 & 0 & 12 \\
5 & & &
\end{tabular}

Analisou-se, também, a relação entre os cinco grupos formados pela análise de agrupamento com as variáveis dendrométricas obtidas, verificando se os grupos formados explicam a variação de produtividade da área do experimento. Para essa análise, foi utilizada a ANOVA, na qual os grupos formados foram definidos como tratamento e as parcelas que pertenceram a cada grupo como repetições.

As variáveis $\mathrm{H}_{\mathrm{dom}}, \mathrm{G} / \mathrm{ha}$ e $\mathrm{V} / \mathrm{ha}$ foram analisadas e pôde-se constatar que para a variável $\mathrm{H}_{\mathrm{dom}}$ não houve diferença estatisticamente significativa entre os grupos, enquanto que para as variáveis $\mathrm{G} / \mathrm{ha}$ e V/ha houve diferença, tendo o teste de Tukey mostrado que apenas o grupo 4 foi inferior aos demais (Tabela 8).

Tabela 8. Teste de Tukey para os 5 grupos formados.

Table 8. Tukey test for 5 groups.

\begin{tabular}{lcccccccc}
\hline Grupos & $\mathbf{H}_{\text {dom }}(\mathbf{m})$ & Tukey & Grupos & $\mathbf{G ~}\left(\mathbf{m}^{2} / \mathbf{h a}\right)$ & Tukey & Grupos & Vol $\left(\mathbf{m}^{\mathbf{3}} \mathbf{h a}\right)$ & Tukey \\
\hline 3 & 21,21 & a & 2 & 57,40 & a & 3 & 550,66 & a \\
5 & 21,13 & a & 3 & 56,96 & a & 2 & 544,52 & a \\
2 & 20,99 & a & 1 & 56,83 & a & 1 & 537,05 & a \\
1 & 20,47 & a & 5 & 54,79 & ab & 5 & 510,14 & ab \\
4 & 18,92 & a & 4 & 39,28 & b & 4 & 350,37 & b \\
\hline
\end{tabular}

Observa-se que o grupo 3 apresentou uma pequena superioridade em relação aos demais, e o grupo 4 teve uma notória diferença de produtividade, apresentando valores bastante inferiores aos dos demais grupos. 
Para $\mathrm{H}_{\mathrm{dom}}$, pode-se observar a inexistência de diferenças significativas entre os grupos. Considerando-se essa variável como a que melhor representa a capacidade produtiva, tal resultado permite afirmar que a altura dominante não conseguiu explicar a variação das características do solo.

Para G/ha e V/ha constatou-se que o grupo 4 foi inferior aos demais, o que permite inferir que, neste estudo, o fator solo foi considerado um fator limitante para o crescimento do pinheiro-do-paraná apenas em solos com baixa nutrição e más características físicas. Características médias e boas de solos aparentemente não acarretam diferenças na produção. Este resultado pode explicar a baixa correlação entre as variáveis de solo e as dendrométricas, significando que a alteração na produção não se dará, necessariamente, por aumento ou falta de nutrientes.

Pode-se observar que, para o grupo 4 (formado pelas parcelas 6, 7 e 8), a profundidade de solo foi menor que um (1) metro. Considerando que essa variável apresentou maiores correlações quando comparada com as demais e que também foi a principal variável na análise discriminante dos grupos, pode-se afirmar, então, que há dois sítios distintos no local do experimento, sendo o primeiro formado pelas parcelas do grupo 1, 2, 3 e 5, e outro, de menor produtividade, formado pelo grupo 4, com solos com profundidade menor que $1 \mathrm{~m}$ definindo a classe de sítio em que o experimento está contido.

\section{CONCLUSÕES}

A análise dos resultados obtidos neste estudo permitiu concluir que:

- Não houve diferença de produtividade das árvores entre as 12 procedências de Araucaria angustifolia.

- A análise do fator solo como fonte da variação de sítio permitiu constatar baixa correlação entre a altura dominante e as variáveis edáficas, tendo os dados de morfologia do solo apresentado maior correlação com a altura dominante, seguidos das variáveis físicas e químicas.

- As variáveis morfológicas, físicas e químicas do solo explicam, respectivamente, $45 \%, 18,1 \%$ e $10,5 \%$ da variação do sítio.

- Foram determinados cinco grupos de parcelas em função de características de solos, sendo mais importantes, em ordem decrescente, as variáveis profundidade, densidade, macroporosidade, areia fina, nitrogênio e cálcio.

- As variáveis $\left(\mathrm{H}_{\mathrm{dom}}, \mathrm{G} / \mathrm{ha}\right.$ e $\left.\mathrm{V} / \mathrm{ha}\right)$ não refletiram a variação de solo no experimento, ou seja, não foram encontradas diferenças significativas entre as variáveis dendrométricas correspondentes às parcelas das diferentes classes de solo, sendo que apenas três parcelas contíguas foram estatisticamente diferentes das demais para as variáveis $\mathrm{G} / \mathrm{ha}$ e $\mathrm{V} / \mathrm{ha}$.

- Obteve-se para este experimento apenas duas classes de sítio, sendo o pior deles representado pelas parcelas localizadas sobre solos com profundidades menores que $1 \mathrm{~m}$, declividades superiores a $10 \%$ e com más características químicas e físicas.

\section{REFERÊNCIAS}

AGÊNCIA DE INFORMAÇÃO EMBRAPA. Latossolos. Disponível em: <http://www.agencia.cnptia. embrapa.br/Agencia16/AG01/arvore/AG01_96_10112005101956.html>. Acesso em: 15/05/2009.

ALVES, S. T. Estudos de sistemas silviculturais utilizando procedências e progênies de Araucaria angustifolia (Bert.) O. Ktze. Dissertação (Mestrado em Ciências Florestais) - Setor de Ciências Agrárias, Universidade Federal do Paraná, Curitiba, 1985.

BALDANZI, G.; ARAUJO, A. J. de Investigação sobre a variação geográfica na Araucaria angustifolia, na Estação de Pesquisas Florestais de Rio PR. Revista Floresta, Curitiba, v. 3, n. 1. p. 37 - 42, 1971.

BREPOHL, D. A contribuição econômica da exploração da Araucaria angustifolia (Bert.) O. Ktze. In: IUFRO MEETING ON FORESTRY PROBLEMS OF THE GENUS ARAUCARIA. (1, 1979, Curitiba). Forestry problems of genus Araucaria. Curitiba: FUPEF, 1980. p. 347-350

CARVALHO, P. E. R. Espécies arbóreas brasileiras. Brasília: Embrapa Informação Tecnológica, Colombo, 2003. 1039 p. 
CASSOL, C. A. Relações entre características do solo, crescimento e produtividade em povoamentos implantados de Araucaria angustifolia (Bert.) O. Ktze. em Passo Fundo - RS. Dissertação (Mestrado em Ciências Florestais) - Setor de Ciências Agrárias, Universidade Federal do Paraná, Curitiba, 1982.

DE HOOGH, R. J.; DIETRICH, A. B. Avaliação de sítios para Araucária angustifolia (Bert.) O. Ktze. em povoamentos artificiais. Brasil Florestal, Brasília, DF, v. 10, n. 37, p. 19-71, 1979.

DIETRICH, A. B. Relações em dados analíticos de solo: Análise foliar e dados de crescimento da Araucaria angustifolia (Bert.) O. Ktze. 87 p. Dissertação (Mestrado em Ciências Florestais) - Setor de Ciências Agrárias, Universidade Federal do Paraná, Curitiba, 1979.

FAHLER, J. C.; DI LUCCA, C. M. Variación geográfica da Araucaria angustifolia (Bert.) O. Ktze. informe preliminar a los 5 años. In: IUFRO MEETING ON FORESTRY PROBLEMS OF THE GENUS ARAUCARIA (1., 1979, Curitiba). Forestry problems of genus Araucaria. Curitiba: FUPEF, 1980, p. 96-102.

FUNDAÇÃO DE PESQUISAS FLORESTAIS (FUPEF). Inventário florestal do Pinheiro do no sul do Brasil: relatório final. Curitiba, 1978. 327 p.

GERHARD, E. J.; FINGER, C. A. G.; LONGHI, S. J.; SCHUMACHER, M. V. Contribuição da análise multivariada na classificação de sítios em povoamentos de Araucaria angustifolia (Bert.) O. Ktze. baseado nos fatores físicos e morfológicos do solo e no conteúdo de nutrientes da serapilheira. Ciência Florestal, Santa Maria, v. 11, n. 2, p. 41-57, 2001.

GURGEL FILHO, O. T. Silvicultura da Araucaria angustifolia (Bert.) O. Ktze. In: IUFRO MEETING ON FORESTRY PROBLEMS OF THE GENUS ARAUCARIA (1., 1979, Curitiba). Forestry problems of genus Araucaria. Curitiba: FUPEF, 1980. p. 96-102.

GURGEL, J. T. A.; GURGEL FILHO, O. T. Evidências de raças geográficas no pinheiro Brasileiro. Araucaria angustifolia (Bert.) O. Ktze. Ciência e Cultura, São Paulo, v. 17, n. 01, 1965 p. 33-39.

HOPPE, J. M. Relações entre dados analíticos do solo, análise foliar e dados de incremento da Araucaria angustifolia (Bert.) O. Ktze., na Floresta Nacional de Passo Fundo, RS. Dissertação (Mestrado em Ciências Florestais) - Setor de Ciências Agrárias, Universidade Federal do Paraná, Curitiba, 1980.

KAGEYAMA, D. Y.; JACOB, A. Variação genética entre e dentro de populações de Araucaria angustifolia (Bert.) O. Ktze. na região de Itapeva - SP, estimada até o $6^{\circ}$ ano de idade In: IUFRO MEETING ON FORESTRY PROBLEMS OF THE GENUS ARAUCARIA (1., 1979, Curitiba). Forestry problems of genus Araucaria. Curitiba: FUPEF, 1980. p. 83- 87.

LORENZI, H. Árvores Brasileiras: manual de identificação e cultivo de plantas arbóreas e nativas do Brasil. Nova Odessa: Plantarum, 1992. 352 p.

MAACK, R. Geografia física do estado do Paraná. Rio de Janeiro: J. Olympio,1981, 450 p.

MONTEIRO, R. F. R.; SPELTZ, R. M. Ensaio de 24 procedências de Araucaria angustifolia (Bert.) O. Ktze. In: IUFRO MEETING ON FORESTRY PROBLEMS OF THE GENUS ARAUCARIA (1., 1979, Curitiba). Forestry problems of genus Araucaria. Curitiba: FUPEF, 1980. p. 181-201.

PRODAN, M.; PETERS, R.; COX, F.; REAL, P. Mensura Forestal. San José, CR: Instituto Interamericano de Cooperación para la Agricultura, 1997, 501 p.

SALLE, G. L. Influência dos fatores ambientais na classificação de sítio para Pinus taeda L., na região de Cambará do Sul, RS, Brasil. 85 p. Dissertação (Mestrado em Engenharia Florestal) Universidade Federal de Santa Maria, Santa Maria, 1993.

SHIMIZU, J. Y.; HIGA, A. R. Variação genética entre procedências de Araucaria angustifolia (Bert.) O. Ktze na região de Itapeva-SP, estimada até o $6^{\circ}$ ano de idade In: IUFRO IUFRO MEETING ON FORESTRY PROBLEMS OF THE GENUS ARAUCARIA (1., 1979, Curitiba). Forestry problems of genus Araucaria. Curitiba: FUPEF, 1980. p. 78-83. 
SIMÕES, J. W. Efeitos da omissão de nutrientes na alimentação mineral do Pinheiro do Paraná Araucaria angustifolia (Bert.) O. Ktze. cultivado em vaso. Tese (Doutorado em Ciências Florestais) Universidade de São Paulo, Piracicaba SP, 1972.

SPURR, S. H. Forest inventory. New York: Ronald, 1952. 476 p.

VAN GOOR, C. P. Classificação da capacidade da terra em relação ao reflorestamento com Pinus elliottii Eng. Var. elliottii e Araucaria angustifolia (Bert.) O. Ktze., no estado de São Pulo. Silvicultura em São Paulo, São Paulo, v. 4, p. 349-366, 1965. 